\title{
Colloidal stability of aqueous suspensions of barium zirconate
}

\author{
Frédéric Boschini ${ }^{\mathrm{a}}$, A. Rulmont ${ }^{\mathrm{a}}$, R. Cloots ${ }^{\mathrm{a}}$, R. Moreno ${ }^{\mathrm{b}, *}$ \\ ${ }^{a}$ Laboratoire de Chimie Inorganique Structurale, Department of Chemistry, University of Liège, B-4000 Liège, Belgium \\ ${ }^{\mathrm{b}}$ Instituto de Cerámica y Vidrio, Consejo Superior de Investigaciones Cientificas (CSIC), Campus de Cantoblanco, E-28049 Madrid, Spain
}

Received 21 February 2004; received in revised form 29 June 2004; accepted 10 July 2004

Available online 11 September 2004

\begin{abstract}
In this article, the colloidal behaviour of aqueous suspensions of barium zirconate is investigated. The variation of $\zeta$-potential as a consequence of changing the $\mathrm{pH}$ and the concentration of an anionic polyelectrolyte is studied, the isoelectric point occurring at $\mathrm{pH}$ 5.3. The IEP shifts down on calcining the powder and also when anionic polyelectrolytes are added. Rheological studies have been made on suspensions prepared to a solids loading of $27 \mathrm{vol} . \%(72 \mathrm{wt} . \%)$. Optimum dispersing conditions are reached for suspensions prepared at basic $\mathrm{pH}$ with $1.5 \mathrm{wt} . \%$ tetramethylammonium hydroxide (TMAH) and a concentration of ammonium polymethacrylate (PMAA) of $1.6 \mathrm{wt} . \%$. Comparison with similar suspensions prepared with $\mathrm{NaOH}$ instead PMAA demonstrated that TMAH gives an extra contribution to stability, probably related to the adsorption of $\mathrm{N}^{+}(\mathrm{Me})_{4}$ groups. The suspensions were slip cast, and green densities higher than $60 \%$ of theoretical were obtained. (C) 2004 Elsevier Ltd. All rights reserved.
\end{abstract}

Keywords: Suspensions; Colloidal behaviour; Rheology; Slip casting; $\mathrm{BaZrO}_{3}$

\section{Introduction}

Barium zirconate $\left(\mathrm{BaZrO}_{3}\right)$ is a very promising refractory structural material with a very high melting point $\left(2600^{\circ} \mathrm{C}\right)$. It is used extensively in the fabrication of crucibles for the synthesis of superconductors at high temperature, ${ }^{1,2}$ because of its high resistance to chemical attack by highly corrosive molten phases, as those appearing during the synthesis of superconductors. For such application, high density ceramic parts are requested to avoid the percolation of the melting flux through the crucible. However, denosification of $\mathrm{BaZrO}_{3}$ to $97 \%$ of the theoretical density needs a thermal treatment at high temperature $\left(1700^{\circ} \mathrm{C}\right)$ and long sintering times $(48 \mathrm{~h}) .{ }^{1}$

Hence, the goal is to produce $\mathrm{BaZrO}_{3}$ pieces with full density. Much effort has been devoted recently to study the synthesis of barium zirconate powders with enhanced sinterability to obtain dense ceramic parts at lower temperature. A variety of synthesis methods have been developed, such

\footnotetext{
* Corresponding author. Tel.: +34 91 7355849; fax: +34 917355843.

E-mail address: rmoreno@icv.csic.es (R. Moreno).
}

as the citrate route, the oxalate route ${ }^{3,4}$ or the metal-chelate sol-gel route using ethylene glycol as complexing agent. ${ }^{5,6}$

A second feature is to establish a controlled processing route to optimise the manufacturing process. The uniformity and the microstructure of the sintered body are strongly dependent on the shape forming technique selected for its production. However, no studies on the shape forming of barium zirconate have been found in the literature. Barium zirconate parts are available in the market and they are usually produced by isostatic pressing.

Among the shape forming processes, slip casting has demonstrated to be very simple and highly efficient for obtaining complex parts with controlled microstructure and high reliability. A key point in slip casting is to achieve a welldispersed suspension with moderate to high volume fraction of particles. The dispersion of $\mathrm{BaZrO}_{3}$ powders in water has not yet been described in the literature. However, much effort has been made to understand the dispersion of $\mathrm{BaTiO}_{3}$, which has similar chemistry than $\mathrm{BaZrO}_{3}$.

Barium titanate is used in the production of multilayer ceramic capacitors, which are usually prepared by tape casting. 
The suspending media are preferentially organic solvents, but recently, an attempt has been made to replace nonaqueousby aqueous-based suspensions.

The colloidal properties of aqueous barium titanate suspensions are complex and not fully understood. According to Blanco-López et al. ${ }^{7}$ the true isoelectric point (IEP) of $\mathrm{BaTiO}_{3}$ is 6.7 , but it is strongly dependent on $\mathrm{Ba}^{2+}$ concentration and thus on the contamination by $\mathrm{BaCO}_{3}$, always present as impurity phase. They state that it is possible to deflocculate $\mathrm{BaTiO}_{3}$ aqueous slip by reducing the $\mathrm{pH}$ to 3-4. Anderson et al. ${ }^{8}$ demonstrated that $\mathrm{Ba}$ dissolution increases with decreasing $\mathrm{pH}$. Hence, at acidic $\mathrm{pH}$ an excess of $\mathrm{Ba}^{2+}$ ions can promote flocculation and concentrated suspensions may have too high viscosities, which result in low green densities. Paik et al. ${ }^{9}$ have studied the complex electrokinetic behaviour of barium titanate aqueous suspensions as a function of $\mathrm{pH}$, suggesting that inherent $\mathrm{BaCO}_{3}$ contamination is not the primary source of dissolved $\mathrm{Ba}$.

The dispersion of concentrated aqueous suspensions of $\mathrm{BaTiO}_{3}$ has been studied using cationic and anionic polyelectrolytes. Cationic polyelectrolytes were expected to be more adequate because their active group was indifferent to $\mathrm{Ba}^{2+}$ ions, ${ }^{10}$ but experimental results demonstrated that cationic polymers were not very effective for the deflocculation. ${ }^{11-13}$ On the other hand, anionic polyelectrolytes seem to be suitable for the deflocculation of this system. In particular, ammonium polyacrylates have been shown to be very effective deflocculants, specially in the $\mathrm{pH}$ range 7-12, where the amount of dissolved $\mathrm{Ba}$ is lower and PAA is negatively charged (like barium titanate) and adsorbs to the surface through the minority of positive surface sites of $\mathrm{Ba}^{2+} .14$

Since from a chemical point of view, $\mathrm{BaZrO}_{3}$ is very similar to $\mathrm{BaTiO}_{3}$, a similar colloid chemistry is also expected. Thus, dispersion of both powders in water should present some common points, like dissolution at acidic $\mathrm{pHs}$ and complex electrokinetic behaviour. From the published studies on the aqueous processing for $\mathrm{BaTiO}_{3}$ one might expect that $\mathrm{BaZrO}_{3}$ should be efficiently dispersed with anionic polyelectrolytes at basic $\mathrm{pH}$ conditions.

This work aims to study the colloidal properties of $\mathrm{BaZrO}_{3}$ aqueous suspensions by means of $\zeta$-potential measurements and rheological characterisation, focusing on the combined effect of $\mathrm{pH}$ and polyelectrolytes.

\section{Experimental procedure}

\subsection{Materials}

The barium zirconate used in this study (Alfa Aesar, 99\% purity) was commercial powder produced by solid state reaction between $\mathrm{BaCO}_{3}$ and $\mathrm{ZrO}_{2}$. This powder has a specific surface area (BET) of $3.5 \mathrm{~m}^{2} / \mathrm{g}$ and a particle size around $1.5 \mu \mathrm{m}$. For this study, the powder was milled in ethanol with a planetary mill with zirconia balls to reach a median particle size around $0.5 \mu \mathrm{m}$ and a surface area (BET) of $11.2 \mathrm{~m}^{2} / \mathrm{g}$.

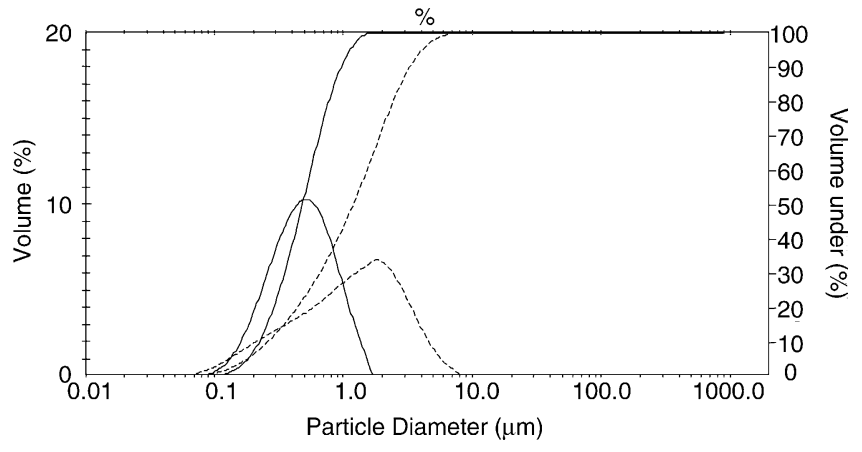

Fig. 1. Particle size distribution of the $\mathrm{BaZrO}_{3}$ powder: as-received (dashed line), and after milling in ethanol (continuous line).

Particle size distribution curves of the unmilled and the milled barium zirconate powder are shown in Fig. 1. Fig. 2(a) and (b) show microphotographs of the as-received and the milled powder, respectively. The milling process leads to deagglomeration of the as-received powder. All experiments reported in this work were performed using the milled powder.

An ammonium polymethacrylate (Dolapix CE 64, Zschimmer \& Schwarz, Germany) was used as deflocculant. This deflocculant is supplied as a solution with a concentration of active matter of $35 \%$. Concentrations are expressed as weight percent (wt.\%), referred to the solids loading, of the as-received product. Basicity was provided by adding quaternary ammonium hydroxides ${ }^{15}$, mainly tetramethylammonium hydroxide (TMAH), although tetraethylammonium hydroxide (TEAH) and tetrapropylammonium hydroxide (TPAH) were also tested. All of them were supplied by Aldrich-Chemie (Germany). These products are supplied as aqueous solutions with different concentrations. The concentration of the bases is expressed also as wt.\% of as-received product, referred to the solids loading of ceramic powder.

\subsection{Measurements and analysis}

The $\zeta$-potential of the $\mathrm{BaZrO}_{3}$ particles were measured with a micro-electrophoresis equipment (Zeta-Meter system 3.0, USA). Measurements were preformed on dilute suspensions prepared with $0.05 \mathrm{~g}$ of $\mathrm{BaZrO}_{3}$ for 11 of pure deionised water. The ionic strength was maintained constant at $10^{-3} \mathrm{M}$ using $\mathrm{KCl}$ as indifferent electrolyte. $\mathrm{pH}$ adjustments were made by adding appropriate amounts of $\mathrm{HCl}$ and $\mathrm{KOH}\left(10^{-2} \mathrm{M}\right)$. The variation of $\zeta$-potential with added polyelectrolyte was measured at the same conditions. In this case $\mathrm{pH}$ was fixed to 9 or 10 by adding TMAH. A fraction of the powder was calcined at $800^{\circ} \mathrm{C} / 12 \mathrm{~h}$ and used also for $\zeta$-potential measurements.

$\mathrm{BaZrO}_{3}$ dissolution at various $\mathrm{pH}$ values was determined by measuring the dissolved Ba concentration using an inductively coupled plasma-atomic emission spectroscopy (ICPAES) equipment. Suspensions for this analysis were prepared to a concentration of $3 \mathrm{vol} . \%$ at the selected $\mathrm{pH}$ conditions. After homogenising with ultrasounds, they were left for equi- 

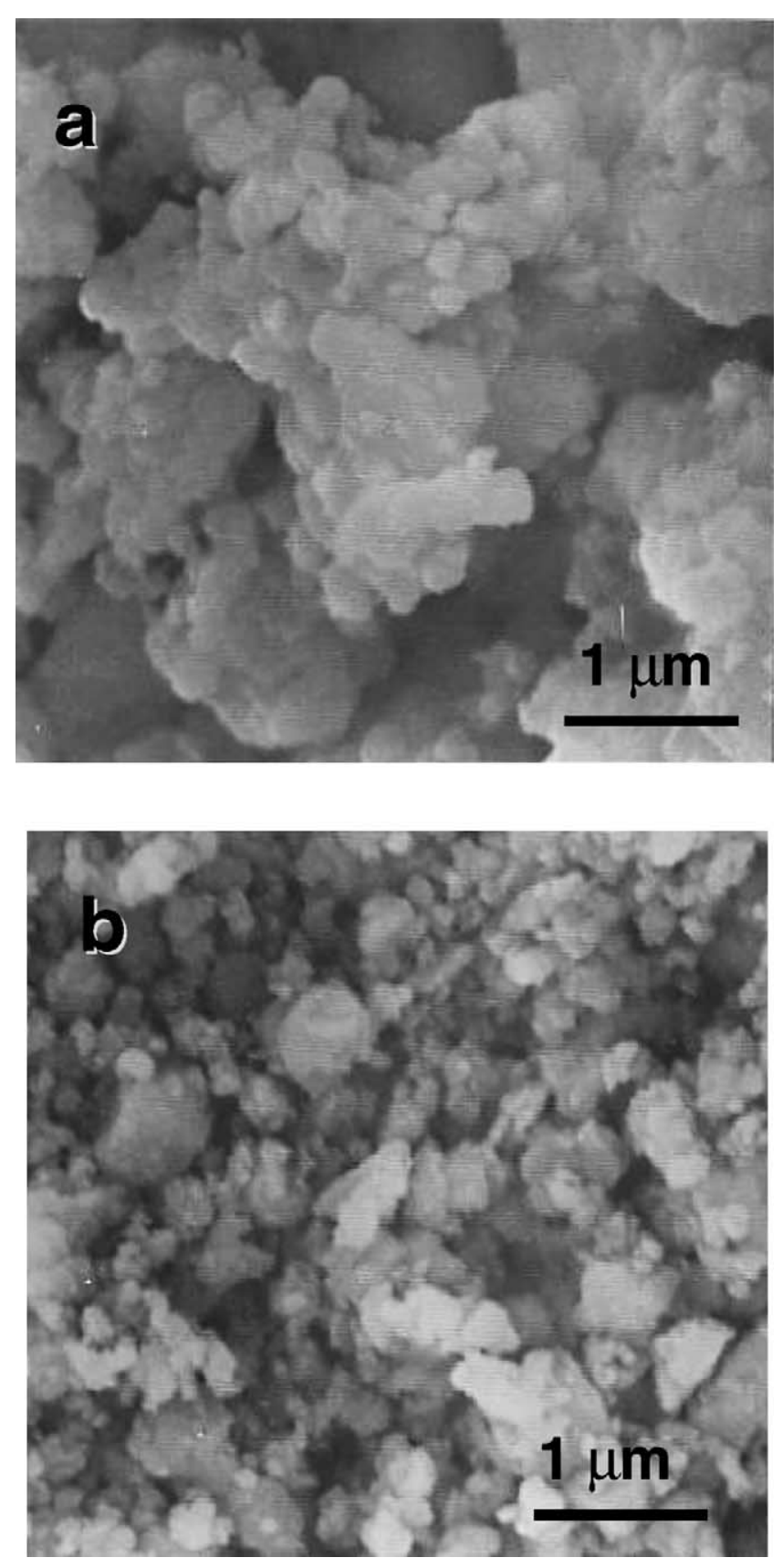

Fig. 2. SEM micrographs of the $\mathrm{BaZrO}_{3}$ powder: as-received (a) and after milling in ethanol (b).

libration period of $48 \mathrm{~h}$ and then centrifuged. The clear supernatant was used for ICP-AES analysis.

Slurries with high solids content ( 29 vol.\%, i.e. $72 \mathrm{wt} . \%)$ were used for the rheological studies. Suspensions were prepared by adding an appropriate amount of powder into the deionised water containing the desired amount of polyelectrolytes to obtain a total volume of $25 \mathrm{ml}$. All slips were deagglomerated with a high energy ultrasonic probe (IKA 400S, Germany) for $1 \mathrm{~min}$ and stirred for $20 \mathrm{~h}$ before measurements to establish an equilibrium dispersion system.
The rheological behaviour of all studied slips was determined using a rheometer (Haake RS50, Karlsruhe, Germany) capable to operate at both controlled shear rate (CR) and controlled shear stress (CS) conditions. The first mode was used to measure the flow curves, and the second for determining the yield values, calculated from the double logarithmic plots of deformation versus shear stress. The measurements were performed using a double cone and plate system, with a solvent trap, at a constant temperature of $25^{\circ} \mathrm{C}$.

Small ceramic discs with $2 \mathrm{~cm}$ in diameter were obtained by slip casting suspensions prepared at different conditions (concentrations of polyelectrolyte and quaternary ammonium hydroxide) on plaster of Paris moulds. The green density of the disc-shaped specimens was measured by mercury immersion after drying for $48 \mathrm{~h}$ at room conditions and the values are reported as percentage of theoretical density of $\mathrm{BaZrO}_{3}$ $\left(6.23 \mathrm{~g} / \mathrm{cm}^{3}\right)$.

\section{Results and discussion}

\section{1. $\zeta$-Potential of $\mathrm{BaZrO}_{3}$ suspensions}

It has been stated elsewhere that when barium titanate powder is in contact with water at acidic $\mathrm{pH}$ conditions a surface dissolution of $\mathrm{Ba}^{2+}$ ions occurs, and the particle surface shows an increasing $\mathrm{TiO}_{2}$-like behaviour as $\mathrm{pH}$ decreases. A similar behaviour is expected to occur in aqueous suspensions of $\mathrm{BaZrO}_{3}$. The chemical analysis performed by ICP-AES reveals that the powder has a $\mathrm{Ba} / \mathrm{Zr}$ ratio of 0.97 , which maintains constant after milling. Fig. 3 shows the concentration of dissolved $\mathrm{Ba}^{2+}$ ions as a function of $\mathrm{pH}$. This curve clearly shows that there is a drastic increase of the concentration of $\mathrm{Ba}^{2+}$ ions when the $\mathrm{pH}$ of the solution becomes more acid. Thus, $\mathrm{BaZrO}_{3}$ powder presents a similar behaviour as $\mathrm{BaTiO}_{3}$. Therefore, it is better to work at high $\mathrm{pH}$ to reduce $\mathrm{Ba}^{2+}$ dissolution, which can lead to significant changes of the surface properties of the particle. Moreover, barium/zirconium ratio has to be controlled to avoid reaction between the barium zirconate substrate and highly corrosive flux of superconductors at high temperature.

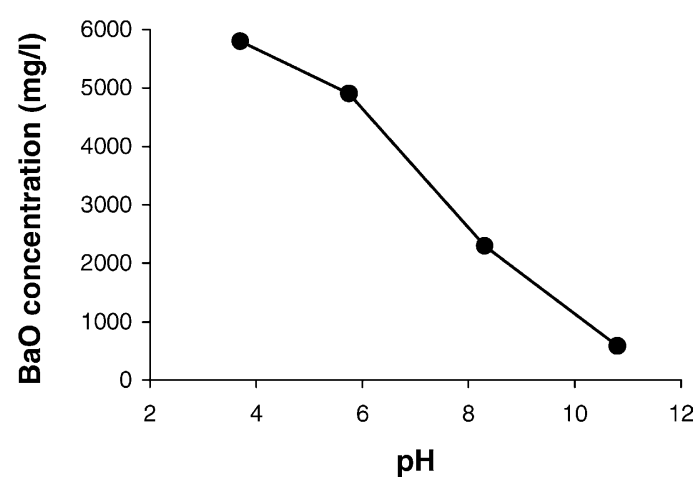

Fig. 3. Evolution of dissolved $\mathrm{Ba}^{2+}$ as a function of $\mathrm{pH}$. 


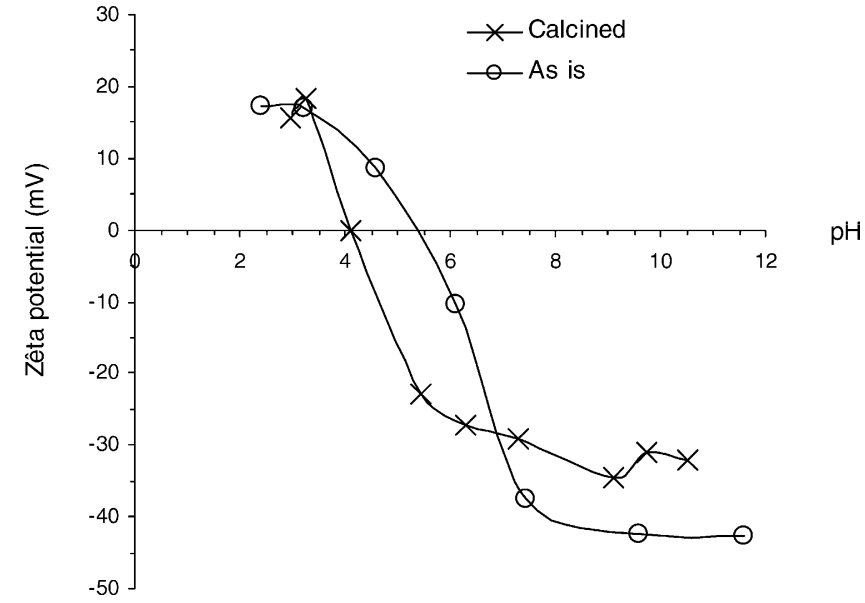

Fig. 4. Variation of $\zeta$-potential with $\mathrm{pH}$ for the milled powder and after calcinations.

Suspension stability as a function of $\mathrm{pH}$ was studied by $\zeta$-potential measurements. The results are shown in Fig. 4. The isoelectric point (IEP) of $\mathrm{BaZrO}_{3}$ particles occurs at $\mathrm{pH}$ $\sim 5.5$, one unity of $\mathrm{pH}$ below that found for $\mathrm{BaTiO}_{3}$ measured on similarly diluted suspensions. ${ }^{7}$ The figure shows that the largest values of $\zeta$-potential and thus, maximum stability, occurs at alkaline $\mathrm{pH}$ values; a value of $\zeta \sim-37.5 \mathrm{mV}$ is obtained at $\mathrm{pH}$ 7.5. The $\zeta$-potential slightly increases as $\mathrm{pH}$ increases, reaching a maximum value of $\sim 42.5 \mathrm{mV}$ in the $\mathrm{pH}$ range 9-12. This is in good agreement with the concentration of dissolved $\mathrm{Ba}^{2+}$ as a function of $\mathrm{pH}$ shown in Fig. 3. Hence, to avoid excessive barium dissolution from the surface of the particles and to reach a value of $\zeta$-potential high enough to assure good suspension stability, dispersion of the particles have to be done in a basic medium.

In Fig. 4, the evolution of $\zeta$-potential with $\mathrm{pH}$ for the calcined powder is also shown. As observed, powder calcinations make the IEP to shift down to $\mathrm{pH} \sim 4$. A similar decrease in the IEP toward acidic values was observed by Blanco-López et al. ${ }^{7}$ which was related to the partial elimination of the surface amphoteric $\mathrm{OH}$ groups.

Ammonium polymethacrylate (PMAA) is a very effective dispersant for the stabilisation of high solids content suspensions. ${ }^{12-14,16}$ This polyelectrolyte is formed by monomer units with a carboxylate function whose dissociation degree depends on the $\mathrm{pH}$ of the solution. ${ }^{17}$ Under basic $\mathrm{pH}$ conditions, a high dissociation degree is reached that enhances adsorption of anionic polymer chains onto the particles surface, thus allowing an efficient dispersion through an electrosteric mechanism. The combined effect of polyelectrolyte and $\mathrm{pH}$ was studied by $\zeta$-potential measurements.

Fig. 5 shows the variation of $\zeta$-potential as a function of $\mathrm{pH}$ for $\mathrm{BaZrO}_{3}$ suspensions ( $\left.0.05 \mathrm{wt} . \%\right)$ containing $1 \mathrm{wt} . \%$ of polyelectrolyte. The isoelectric point shifts below $\mathrm{pH} 3.5$, as expected when anionic polyelectrolytes are adsorbed on the particle surface. In the alkaline region, values of $\zeta$-potential maintain high and similar to those obtained for the powder without deflocculant.

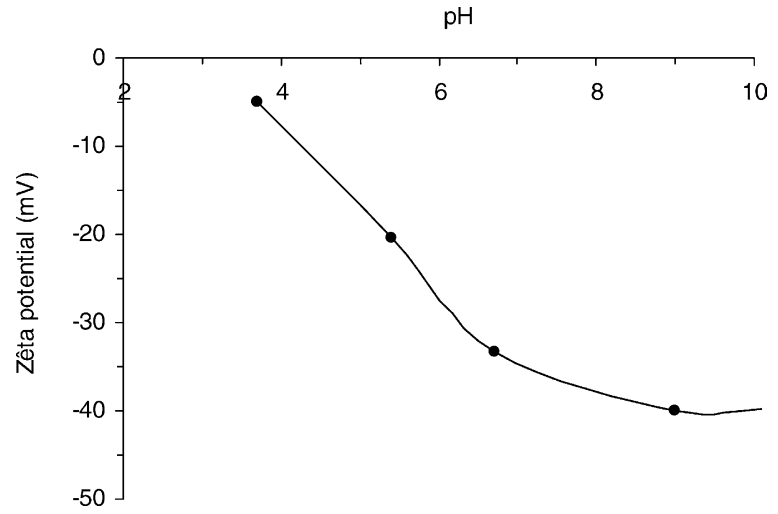

Fig. 5. Variation of $\zeta$-potential vs. $\mathrm{pH}$ for suspensions with $1.0 \mathrm{wt} \%$ polyelectrolyte.

Fig. 6 shows the variation of $\zeta$-potential as a function of polyelectrolyte concentration for suspensions prepared at $\mathrm{pH}$ 9 and 10 and left for $24 \mathrm{~h}$ equilibration time. $\zeta$-potential values increase with increasing $\mathrm{pH}$ for the same polyelectrolyte concentration. For suspensions with $\mathrm{pH} 10$ a value of $-35 \mathrm{mV}$ is reached for $0.4 \mathrm{wt} . \%$ polyelectrolyte, high enough to assure stability. For comparison purposes, Fig. 6 also plots the variation of $\zeta$-potential with polyelectrolyte content for suspensions of the calcined powder prepared at $\mathrm{pH} 9$. The values are rather coincident with those obtained for the untreated

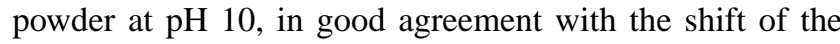
isoelectric point occurring after calcinations.

\subsection{Rheological properties of $\mathrm{BaZrO}_{3}$ suspensions}

High solids content barium titanate suspensions have been successfully prepared using PAA and PMAA at different $\mathrm{pH} .{ }^{10-14}$ We have attempted to prepare concentrated aqueous suspensions of $\mathrm{BaZrO}_{3}$ with a similar anionic polyelectrolyte in a broad range of $\mathrm{pH}$, but viscosity was always high and no stable suspensions were achieved. On the other hand, the use of a strong base (either alkyl ammonium hydroxide or $\mathrm{NaOH}$ ) did not allow itself a good dispersion. To obtain

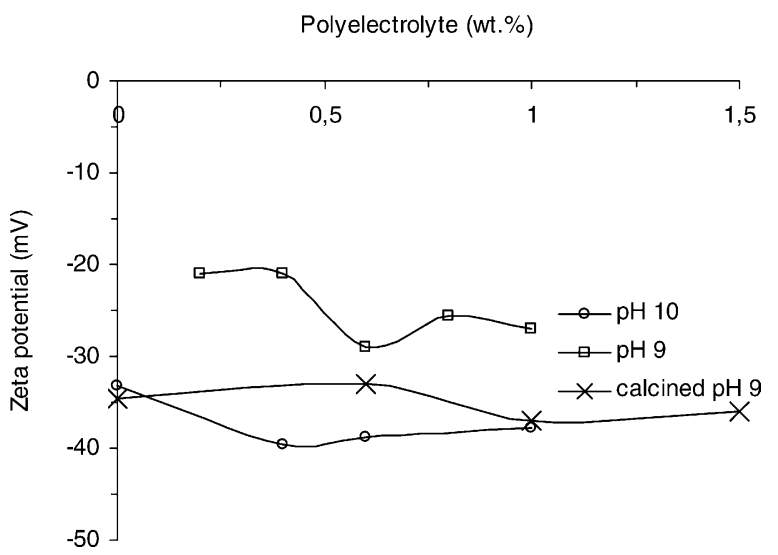

Fig. 6. Variation of $\zeta$-potential vs. polyelectrolyte concentration at $\mathrm{pH} 9$ and 10 , and for the calcined powder at $\mathrm{pH} 10$. 


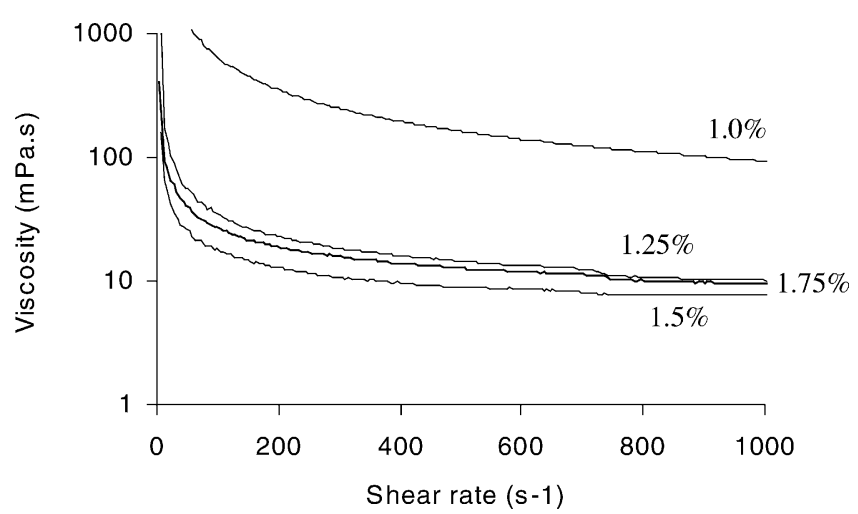

Fig. 7. Viscosity curves of 29 vol. $\%$ suspensions with $1.0 \mathrm{wt} . \%$ polyelectrolyte and different TMAH contents.

a well-dispersed, low-viscosity concentrated suspension of barium zirconate the concourse of both the polyelectrolyte and the base was necessary.

For this purpose, strong organic bases as tetra(methyl,ethyl,propyl)-ammonium hydroxide have been used for $\mathrm{pH}$ adjustments. These bases have two advantages over inorganic strong bases like $\mathrm{NaOH}$ : (1) the contamination of the final ceramic parts by inorganic cations which are potential glass-formers is avoided, and (2) according to previous work on silicon nitride suspensions, TMAH (and so, TEAH and TEPH) are in fact quaternary ammonium surfactants, so that positively charged nitrogen atom can adsorb specifically onto the particle surface, thus enhancing stabilisation. $^{15}$

Hence, to study the rheological properties of concentrated slips of $\mathrm{BaZrO}_{3}(29 \mathrm{vol. \%})$ two sets of suspensions were prepared. Firstly, suspensions were prepared with a constant concentration of polyelectrolyte of $1.0 \mathrm{wt} . \%$ and different concentrations of TMAH. Secondly, suspensions were prepared with a constant content of TMAH of $1.5 \mathrm{wt} . \%$ and polyelectrolyte concentrations of $0.8,1.0,1.6$ and $2.4 \mathrm{wt} . \%$.

Fig. 7 shows the viscosity curves of the slips containing $1.0 \mathrm{wt} . \%$ polyelectrolyte and different amounts of TMAH

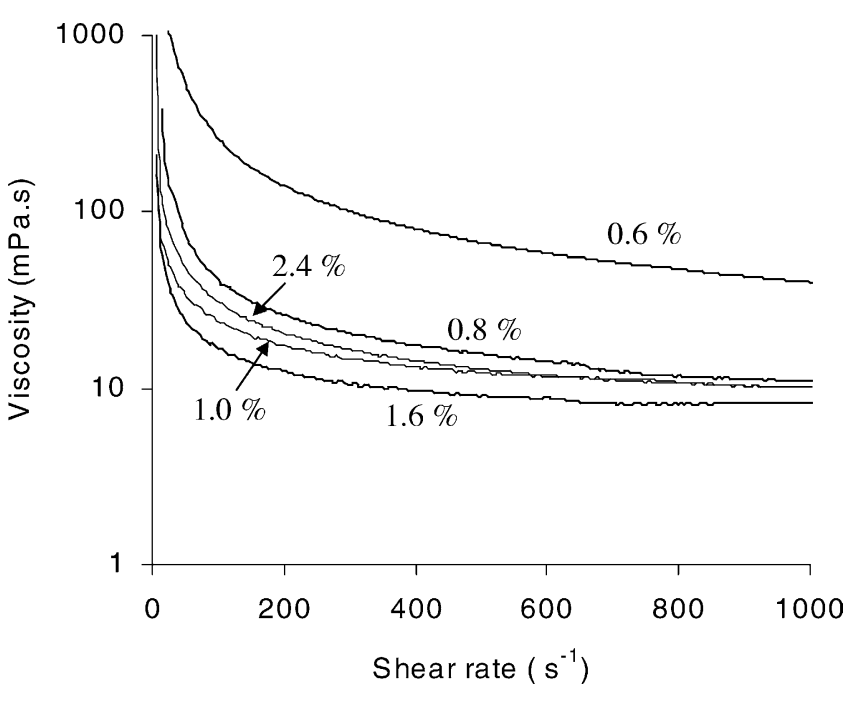

Fig. 8. Viscosity curves of 29 vol.\% suspensions with $1.5 \mathrm{wt} . \%$ TMAH and different polyelectrolyte contents.

measured in controlled rate. All the slips show a plastic Binhgam flow. The suspension containing $1 \mathrm{wt} . \%$ of TMAH is too viscous to be used for slip casting. Viscosity minimum is reached for the slurry containing $1.5 \mathrm{wt}$. $\%$ of TMAH. However, an excess of TMAH does not significantly increase the viscosity. Table 1 summarises the main rheological properties of these suspensions. The viscosity and the thixotropy values were taken from the curves measured in controlled rate mode. The yield stress was calculated as the intercept point of the two straight lines registered in the $\log -\log$ plot of strain versus shear stress measured in controlled stress mode. ${ }^{18}$ All suspensions were slip cast and the resulting relative green density is shown in Table 1, also.

Once the concentration of TMAH was fixed at $1.5 \mathrm{wt} . \%$, the effect of the polyelectrolyte concentration was studied. Fig. 8 shows the viscosity curves of suspensions with different concentrations of polyelectrolyte measured in controlled rate mode. The rheological characteristics of these slips and the green density of the cast bodies are shown in Table 2. The most suitable rheological properties are obtained for

Table 1

Rheological properties of slips with $1 \mathrm{wt}$ \% polyelectrolyte and different TMAH contents and relative density of the resulting green casts

\begin{tabular}{|c|c|c|c|c|}
\hline TMAH (wt.\%) & Yield stress $(\mathrm{Pa})$ & Thixotropy $(\mathrm{Pa} / \mathrm{s})$ & Viscosity at $100 \mathrm{~s}^{-1}$ (mPas) & Green density (\% TD) \\
\hline 1.0 & Impossible & 2495 & 643 & Impossible \\
\hline 1.25 & 2.18 & 1260 & 35 & 50.0 \\
\hline 1.5 & 0.38 & 525 & 18.6 & 55.9 \\
\hline 1.75 & 0.57 & 1063 & 27.2 & 52.5 \\
\hline
\end{tabular}

Table 2

Rheological properties of slips with $1.5 \mathrm{wt} \%$ TMAH and different polyelectrolyte contents and relative density of the resulting green casts

\begin{tabular}{llll}
\hline Polyelectrolyte (wt.\%) & Yield stress (Pa) & Thixotropy (Pa/s) & Viscosity at $100 \mathrm{~s}^{-1}(\mathrm{mPa})$ \\
\hline 0.8 & 6.87 & 1132 & 25.9 \\
1.0 & 0.38 & 526 & 17.1 \\
1.6 & 0.37 & 388 & 12.4 \\
2.4 & 1.22 & 707 & 20.2 \\
\hline
\end{tabular}


Table 3

Rheological properties of slips with $1.6 \mathrm{wt} . \%$ polyelectrolyte and $1.5 \mathrm{wt}$.\% quaternary ammonium with different alkyl chain length, and relative density of the resulting green casts

\begin{tabular}{lllll}
\hline Quaternary ammonium & Yield stress $(\mathrm{Pa})$ & Thixotropy $(\mathrm{Pa} / \mathrm{s})$ & Viscosity at $100 \mathrm{~s}^{-1}(\mathrm{mPa})$ & Green density $(\%$ TD) \\
\hline TMAH & 0.38 & 525 & 18.6 & 55.9 \\
TEAH & 0.44 & 564 & 17.2 & 54.7 \\
TPAH & 0.48 & 656 & 24.7 & 54.1 \\
\hline
\end{tabular}

polyelectrolyte contents of 1.0 and $1.6 \mathrm{wt} . \%$. Yield stresses are very similar, but viscosity is lower for $1.6 \mathrm{wt} . \%$. The highest green density is reached for this polyelectrolyte concentration.

The effect of the length of the alkyl chain of the quaternary ammonium was studied through the rheological behaviour of the slips. Three different tetraalkylammonium hydroxides were added to the slips to the same molar concentration: TMAH, TEAH and TPAH. The viscosity curves of suspensions prepared with $1.6 \mathrm{wt} . \%$ polyelectrolyte and $1.5 \mathrm{wt} . \%$ of each base are plotted in Fig. 9. The rheological properties of these slips and the green density of the cast bodies are compared in Table 3. Viscosities are similar for TMAH and TEAH and increases for TPAH. The yield stress and the thixotropy increase with the chain length. Accordingly, the resulting green density decreases as the chain length increases, thus confirming that TMAH is the most suitable base for stabilising aqueous $\mathrm{BaZrO}_{3}$ suspensions.

TMAH can specifically adsorb on the particles surface giving place to an electrosteric mechanism, as a conventional cationic surfactant. To evaluate this extra contribution to stability, suspensions have been prepared at similar conditions replacing TMAH by $\mathrm{NaOH}$. Fig. 10 shows the flow curves of $\mathrm{BaZrO}_{3}$ suspensions with $1.6 \mathrm{wt} . \%$ polyelectrolyte using either TMAH and $\mathrm{NaOH}$ as strong base. It can be seen that replacing TMAH by $\mathrm{NaOH}$ leads to significantly higher viscosities, which demonstrates that the $\mathrm{pH}$ value itself does not account for the stability of the suspensions. Therefore, the additional contribution to stability of TMAH could be associated to the attachment of $\mathrm{N}^{+}(\mathrm{Me})_{4}$ ions to the particle surface.

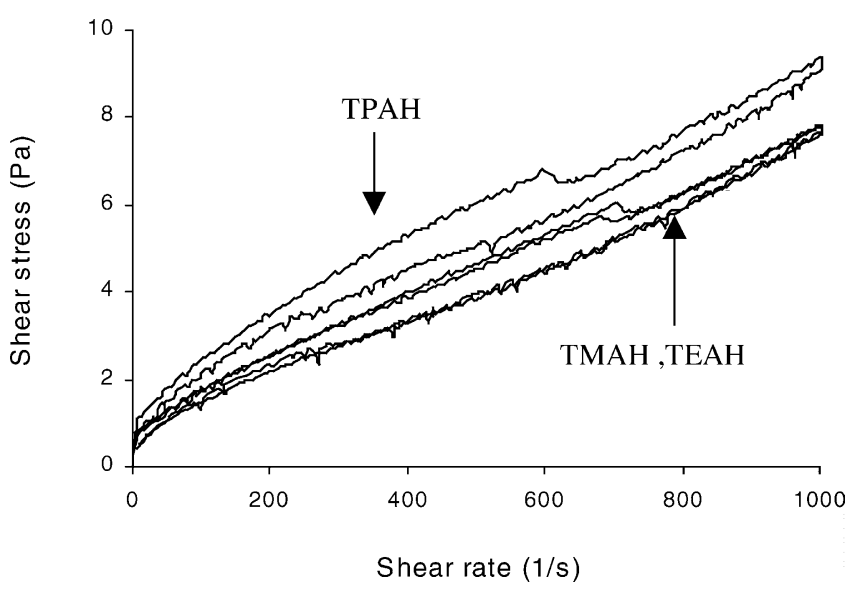

Fig. 9. Effect of the length of the alkyl chain of the quaternary ammonium on the flow curves of $29 \mathrm{vol} . \%$ suspensions with $1.0 \mathrm{wt} . \%$ polyelectrolyte.

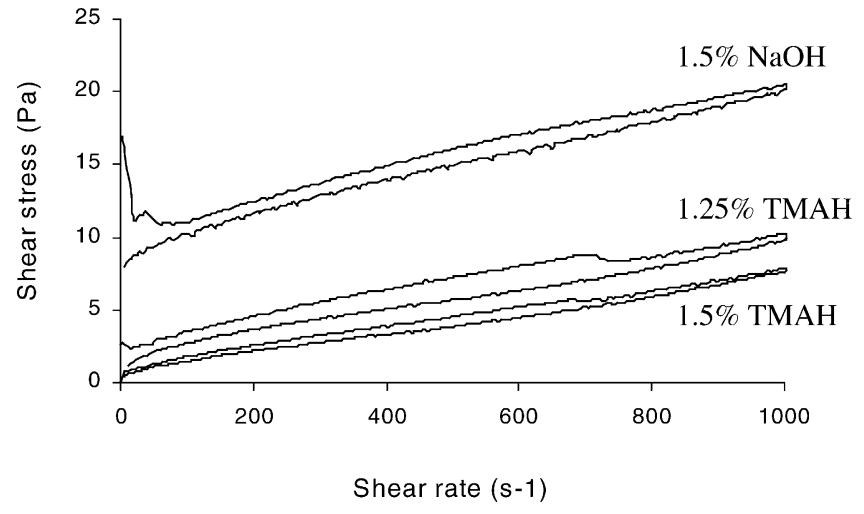

Fig. 10. Effect of the nature of the base in the flow curves of 29 vol.\% suspensions with $1.6 \mathrm{wt} . \%$ polyelectrolyte.

\section{Conclusions}

The colloidal stability of aqueous $\mathrm{BaZrO}_{3}$ suspensions has been studied through $\zeta$-potential and rheological measurements. The colloidal behaviour of $\mathrm{BaZrO}_{3}$ suspensions is rather similar to that of $\mathrm{BaTiO}_{3}$. Noticeable dissolution of $\mathrm{Ba}^{2+}$ occurs at acidic $\mathrm{pH}$, so that suspensions might be prepared at alkaline $\mathrm{pH}$. The isoelectric point of $\mathrm{BaZrO}_{3}$ has been found to occur at $\mathrm{pH} \sim 5.5$, and it decreases when the powder is calcined as a consequence of the elimination of surface $\mathrm{OH}$ groups. The addition of an anionic polyelectrolyte promotes a shift of the isoelectric point toward lower $\mathrm{pH}$.

The preparation of stable, concentrated suspensions required the addition of both a polyelectrolyte and a strong base. TMAH has demonstrated to give lower viscosities than an inorganic base like $\mathrm{NaOH}$ for the same $\mathrm{pH}$. This suggests that TMAH contributes to stability not only by controlling the $\mathrm{pH}$ but also by attaching to the particles surface.

Suspensions with $1.5 \mathrm{wt} . \%$ TMAH and 1.0-1.6wt.\% polyelectrolyte (expressed on as-received products, which means by $1 \mathrm{wt} . \%$ of active matter) presented optimum rheological properties for slip casting. Green densities $>60 \%$ of theoretical have been obtained.

\section{Acknowledgement}

The FNRS (Belgian National Fund for Scientific Research) is gratefully acknowledged for financial support. This work has been supported by CICYT, Spain (contract MAT2003-00836). 


\section{References}

1. Erb, A., Walker, E. and Flukiger, R., BaZrO 3 : the solution for the crucible corrosion problem during the single crystal growth of highTc superconductors $\mathrm{REBa}_{2} \mathrm{Cu}_{3} \mathrm{O}_{7-x}: \mathrm{RE}=\mathrm{Y}$, Pr. Physica $C, 1995$, 245, 245-251.

2. Zhang, J. L. and Evetts, J. E., $\mathrm{BaZrO}_{3}$ and $\mathrm{BaHfO}_{3}$ : preparation, properties and compatibility with $\mathrm{YBa}_{2} \mathrm{Cu}_{3} \mathrm{O}_{7-x}$. J. Mater. Sci., 1994 , 29, 778-785.

3. Taglieri, G., Tersigni, M., Villa, P. L. and Mondelli, C., Synthesis by the citrate route and characterization of $\mathrm{BaZrO}_{3}$, a hightech ceramic oxide: preliminary results. Inorg. Mater., 1999, 1, 103110 .

4. Gangadevi, T., Subba Rao, M. and Narayanan, T. R., Thermal decomposition of zirconyl oxalates I. Barium zirconyl oxalate. J. Thermal Anal., 1980, 19, 312-332.

5. Lee, S. J., Biegalski, M. D. and Kriven, W. M., Barium titanate and barium orthotitanate powders through an ethylene glycol polymerization route. J. Mater. Res., 1999, 14, 3001.

6. Veith, M., Mathur, S., Lecerf, N., Huch, V. and Decker, T., Sol-gel synthesis of nano-scaled $\mathrm{BaTiO}_{3}, \mathrm{BaZrO}_{3}$ and $\mathrm{BaTi}_{0.5} \mathrm{Zr}_{0.5} \mathrm{O}_{3}$ oxides via single-source alkoxide precursors and semi-alkoxide routes. $J$. Sol-gel Sci. Technol., 2000, 15, 145-158.

7. Blanco-López, M. C., Rand, B. and Riley, F. L., The isoelectric point of $\mathrm{BaTiO}_{3}$. J. Eur. Ceram. Soc., 2000, 20, 107-118.

8. Anderson, D. A., Adair, J. H., Miller, D., Biggers, J. V., Shrout, T. R. and Miller, D., Surface chemistry effects on ceramic processing of BaTiO3 powder. In Ceramic Transactions. Vol 1. Ceramic Powder Science II, ed. G. L. Messing, E. R. Fuller and H. Hausner. American Ceramic Society, Westerville, OH, 1988, p. 485-L 492.
9. Paik, U., Lee, S. and Hackley, V. A., Influence of barium dissolution on the electrokinetic properties of colloidal $\mathrm{BaTiO}_{3}$ in an aqueous medium. J. Am. Ceram. Soc., 2003, 86, 1662-1668.

10. Blanco-López, M. C., Fourlaris, G., Rand, B. and Riley, F. L., Characterisation of barium titanate powders: barium carbonate identification. J. Am. Ceram. Soc., 1999, 82, 1777-1786.

11. Blanco-López, M. C., Rand, B. and Riley, F. L., Polymeric stabilisation of aqueous suspensions of barium titanate. Part I: Effect of $\mathrm{pH}$. J. Eur. Ceram. Soc., 2000, 20, 1579-1586.

12. Blanco-López, M. C., Rand, B. and Riley, F. L., Polymeric stabilisation of aqueous suspensions of barium titanate. Part II: Effect of polyelectrolyte concentration. J. Eur. Ceram. Soc., 2000, 20, 1587-1594.

13. Yáñez, C. G., Balmori-Ramírez, H. and Martínez, F., Colloidal processing of $\mathrm{BaTiO}_{3}$ using ammonium polyacrylate as dispersant. $\mathrm{Ce}$ ram. Int., 2000, 26, 609-616.

14. Jean, J. H. and Wang, H. R., Stabilization of aqueous $\mathrm{BaTiO}_{3}$ suspensions with ammonium salt of poly(acrylic acid) at various $\mathrm{pH}$ values. J. Mater. Res., 1998, 13, 2245-2250.

15. Moreno, R., Salomoni, A. and Castanho, S. M., Colloidal filtration of silicon nitride aqueous slips. Part I: Optimization of the slip parameters. J. Eur. Ceram. Soc., 1998, 18, 405-416.

16. Davies, J. and Binner, J. G. P., The role of ammonium polyacrylate in dispersing concentrated alumina suspensions. J. Eur. Ceram. Soc., 2000, 20, 1539-1553.

17. Cesarano III, J. and Aksay, I. A., Processing of highly concentrated aqueous $\alpha-\mathrm{Al}_{2} \mathrm{O}_{3}$ suspensions stabilized with polyelectrolytes. J. Am. Ceram. Soc., 1988, 71, 1062-1067.

18. Gutiérrez, C. A., Sánchez-Herencia, A. J. and Moreno, R., Plástico o pseudoplástico? Métodos de determinación y análisis del punto de fluidez de suspensiones cerámicas. Bol. Soc. Esp. Ceram. Vidr., 2000, 39, 229-235. 